Article

\title{
An Efficient Synthesis of Acenaphtho[1,2-b]indole Derivatives via Domino Reaction
}

\author{
Guo-Ning Zhang ${ }^{1,+}{ }^{+}$, Xia Yuan ${ }^{2,+}$, Weiping Niu ${ }^{1}$, Mei Zhu ${ }^{1}$, Juxian Wang ${ }^{1, *}$ and \\ Yucheng Wang ${ }^{1, *}$ \\ 1 Institute of Medicinal Biotechnology, Chinese Academy of Medical Science and Peking Union Medical \\ College, Beijing 100050, China; raisunny2006@163.com (G.-N.Z.); niuweip@126.com (W.N.); \\ mzhu87@163.com (M.Z.) \\ 2 State Key Laboratory of Natural and Biomimetic Drugs, School of Pharmaceutical Sciences, Peking \\ University, Beijing 100191, China; yuanxia@bjmu.edu.cn \\ * Correspondence: imbjxwang@163.com (J.W.); wyc9999@126.com (Y.W.); Tel.: +86-10-63131053 (J.W.); \\ +86-10-6316-5263 (Y.W.) \\ + Both authors contributed equally to this work.
}

Academic Editor: Roman Dembinski

Received: 22 October 2018; Accepted: 20 November 2018; Published: 21 November 2018

\begin{abstract}
A concise and efficient synthesis of acenaphtho[1,2- $b]$ indole derivatives via the domino reactions of enaminones with acenaphthoquinone catalyzed by L-proline has been developed. This protocol has the advantages of good yields, operational convenience and high regioselectivity.
\end{abstract}

Keywords: acenaphtho[1,2-b]indole; domino reaction; enaminone; acenaphthoquinone; L-proline

\section{Introduction}

The indole skeleton is often considered to be one of the most important and fascinating classes of nitrogen-containing heterocycles, and it is often found in both natural products and biologically active compounds [1]. Many indole derivatives have a wide range of biological activities, including anticancer, antioxidant, anti-inflammatory and anti-HIV effects [2-7]. In addition, various polycyclic indoles are privileged scaffolds in medicinal chemistry and drug discovery [8-11]. As a result of these interesting biological activities, many powerful approaches have been developed for the construction of polycyclic indole moieties [12-18]. However, many of these methods have drawbacks, such as limited availability of starting materials, the use of expensive metal catalysts and the need for harsh reaction conditions. Therefore, developing new and efficient methods for the synthesis of polycyclic indoles and their functionalized derivatives using readily available starting materials is of great importance. Enaminones are commercially available starting materials and have proven to be a useful synthons in the construction of a variety of diverse heterocycles. This synthons has been used in the construction of indole moiety via the condensation with $\alpha, \beta$-dicarbonyl compounds under catalyst-free $[19,20]$ or acidic catalyst [21] conditions.

Domino (cascade) reactions are promising and powerful tools in organic and medical chemistry because of their high atom economy, highly complex and diverse products, efficiency in forming multiple bonds, and environmental friendliness [22]. Consequently, domino reactions have often been used for the construction of complex heterocycles [23-28]. As part of our program to develop new methods for the construction of important heterocycles by domino reactions [29-32], we report herein an efficient synthesis of acenaphtho[1,2- $b$ ]indole derivatives via a domino reaction using L-proline as the catalyst. 


\section{Results and Discussion}

We initially evaluated the domino reaction of enaminone $\mathbf{1 a}$ and acenaphthoquinone (2). The reaction mixture of $\mathbf{1 a}$ and $\mathbf{2}$ (1:1 in mole) was subjected to a variety of different conditions and the results are summarized in Table 1. Target product 3a was obtained in 19\% yield when the reaction was carried out under catalyst-free conditions in ethanol at reflux for $2 \mathrm{~h}$ followed by dehydroxylation catalyzed by acid (Table 1, entry 1). To our delight, when L-proline (10 mol \%) was added, the yield increased to $41 \%$ (Table 1, entry 2). Next several other solvents were evaluated for their ability to improve the yield further. The results indicated that toluene was superior to ethanol, chloroform, THF, 1,4-dioxane, DMF, and water in providing much better results (Table 1, entries 2-8). A number of different catalysts were also evaluated for their catalytic efficiency in this reaction. In all cases, the reaction was carried out with $10 \mathrm{~mol} \%$ of the catalyst in toluene at $80{ }^{\circ} \mathrm{C}$ for $2 \mathrm{~h}$. The results revealed that L-proline provided much better results than $p$-TSA, $S$-phenylalanine, phenylalanine, pyrrolidine, piperidine, benzylamine and dibenzylamine (Table 1, entries 9-15). These results indicated that the presence of both secondary nitrogen and a carboxylic acid group plays a crucial role in the desird catalytic activity.

Table 1. Optimization of the reaction conditions.

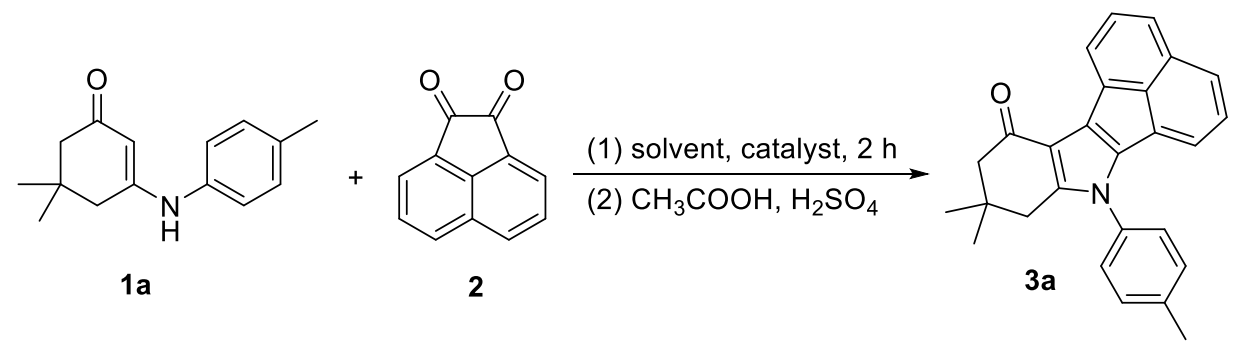

\begin{tabular}{ccccc}
\hline Entry & Solvent & Catalyst (mol \%) & Temperature $\left({ }^{\circ} \mathbf{C}\right)$ & Yield (\%) \\
\hline 1 & Ethanol & No & Reflux & 19 \\
2 & Ethanol & L-Proline (10) & Reflux & 41 \\
3 & Chloroform & L-Proline (10) & Reflux & 28 \\
4 & THF & L-Proline (10) & Reflux & 40 \\
5 & 1,4-Dioxane & L-Proline (10) & Reflux & 23 \\
6 & DMF & L-Proline (10) & 80 & 42 \\
7 & Water & L-Proline (10) & 80 & 20 \\
8 & Toluene & L-Proline (10) & 80 & 65 \\
9 & Toluene & p-TSA (10) & 80 & 46 \\
10 & Toluene & S-Phenylalanine (10) & 80 & 18 \\
11 & Toluene & Phenylamine (10) & 80 & 30 \\
12 & Toluene & Pyrrolidine(10) & 80 & 56 \\
13 & Toluene & Piperidine(10) & 80 & 60 \\
14 & Toluene & Benzylamine(10) & 80 & 40 \\
15 & Toluene & Dibenzylamine(10) & 80 & 63 \\
16 & Toluene & L-Proline (5) & 80 & 45 \\
17 & Toluene & L-Proline (15) & 80 & 59 \\
18 & Toluene & L-Proline (20) & 80 & 45 \\
19 & Toluene & L-Proline (10) & 40 & Trace \\
20 & Toluene & L-Proline (10) & 60 & 25 \\
21 & Toluene & L-Proline (10) & Reflux & 80 \\
\hline
\end{tabular}

Reactions were performed using $\mathbf{1 a}(1 \mathrm{mmol}), 2$ (1 mmol) in solvent $(5 \mathrm{~mL})$.

After L-proline had been identified as the best organocatalyst for this reaction, we decided to test the amount of this catalyst required for the full transformation to the desired compounds. The results revealed that when the amount of L-proline increased from $5 \mathrm{~mol} \%$ to $10 \mathrm{~mol} \%$, the yield also increased from 45 to 65\% (Table 1, entries 16 and 8). The use of $10 \mathrm{~mol} \%$ of L-proline in toluene was effective in pushing this reaction forward, and using larger amounts of the catalyst did not improve the 
yields (Table 1, entries 17-18). The optimization process revealed that the reaction could not proceed in toluene at $40{ }^{\circ} \mathrm{C}$ (Table 1 , entry 19). To identify the optimum reaction temperature, the reaction was conducted in toluene in the presence of $10 \mathrm{~mol} \% \mathrm{~L}$-proline at $60^{\circ} \mathrm{C}, 80^{\circ} \mathrm{C}$, and reflux, and these reactions provided product $3 \mathbf{a}$ in yields of 25,65 and $80 \%$ (Table 1, entries 20, 8 and 21), respectively. On the basis of these results, the optimum reaction condition was identified as refluxing with $10 \mathrm{~mol} \%$ L-proline in toluene for $2 \mathrm{~h}$. Compared with other catalysts (for example, $p$-TSA and TEA), this catalyst has the advantages of higher catalytic efficiency, less toxicity, low cost and ready availability

After the reaction conditions were optimized, the substrate scope of this transformation was also investigated. As shown in Table 2, acenaphthequinone and methyl, bromo, chloro, $t$-Bu and fluoro substituents on the enaminone ring were well tolerated under the reaction conditions, yielding products in satisfactory yields (up to $85 \%$ ). However, when the enaminones with a bulkier group at the 2- or 2- and 6- positions were used, none of the desired products was obtained (Table 2, entries 15-16).

Table 2. Synthesis of acenaphtho[1,2-b]indole derivatives 3 .

\begin{tabular}{cccc} 
\\
\hline
\end{tabular}

The structures of compounds 3 were characterized by IR, ${ }^{1} \mathrm{H}-\mathrm{NMR}$, and ${ }^{13} \mathrm{C}-\mathrm{NMR}$ spectra as well as HRMS. The structure of $\mathbf{3} \mathbf{g}$ was further confirmed using single-crystal X-ray diffraction analysis, (Figure 1).

Although details of the mechanism of the domino reaction remain unclear, the formation of compound 3 could be explained by the reaction sequence shown in Scheme 1. The initial reversible reaction of acenaphthoquinone (2) with L-proline would give iminium ion $\mathbf{A}$. Then, an aza-ene addition of enaminone $\mathbf{1}$ to iminium ion $\mathbf{A}$ leads to intermediate $\mathbf{B}$, which would undergo a rapid tautomerization to give intermediate $\mathbf{C}$. Intermediate $\mathbf{E}$ would be formed by the intramolecular cyclization of intermediate $\mathbf{C}$ and the elimination of L-proline. Then, intermediate $\mathbf{F}$ would be generated by the nucleophilic addition of water to intermediate $\mathbf{E}$. In the last step, product 3 would be formed by dehydroxylation of the intermediate catalyzed by $\mathrm{H}_{2} \mathrm{SO}_{4}$ in acetic acid solution. 


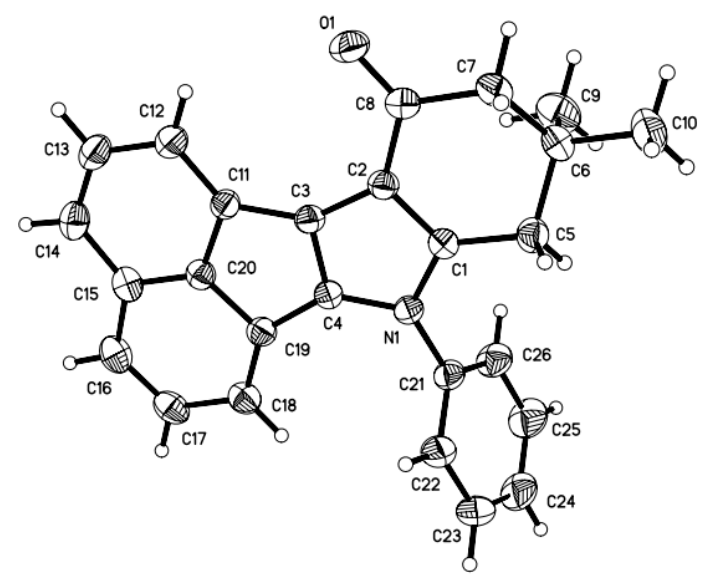

Figure 1. Crystal structure of compound 3g.

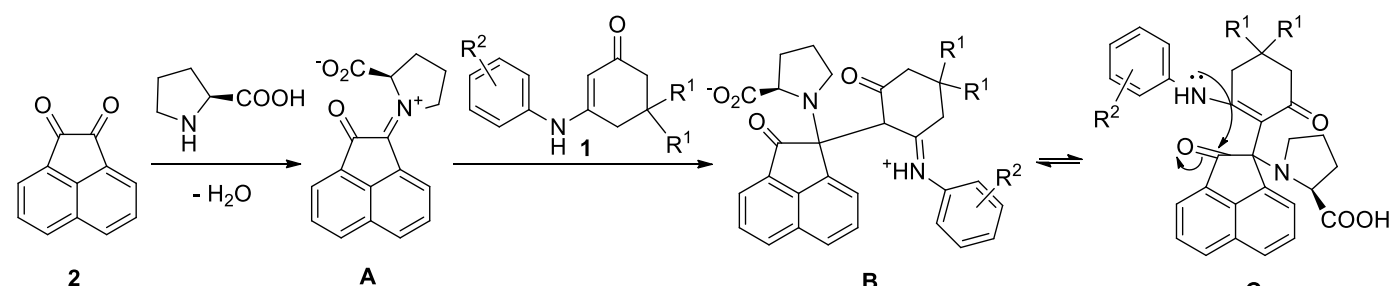

2

A

B

C

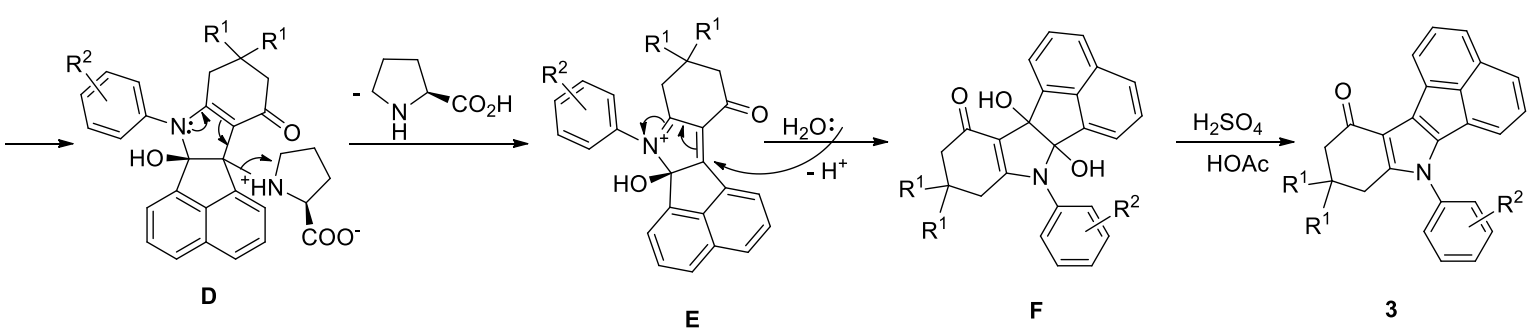

Scheme 1. Proposed mechanism of the synthesis of acenaphtho[1,2-b]indole derivatives 3 .

To support the proposed reaction mechanism, several control experiments were performed (Scheme 2). For example, intermediate $\mathbf{F a}$ was obtained in 84\% yield from the reaction of $\mathbf{1 a}$ with $\mathbf{2}$ in refluxing toluene for $2 \mathrm{~h}$ catalyzed by $10 \mathrm{~mol} \% \mathrm{~L}$-proline. Desired product 3a was obtained in $90 \%$ yield when intermediate $\mathrm{Fa}$ was reacted at $80^{\circ} \mathrm{C}$ for $2 \mathrm{~h}$ in acetic acid catalyzed by $\mathrm{H}_{2} \mathrm{SO}_{4}$.<smiles>Cc1ccc(NC2=CC(=O)CC(C)(C)C2)cc1</smiles>

$1 \mathrm{a}$<smiles>O=C1C(=O)c2cccc3cccc1c23</smiles>

2

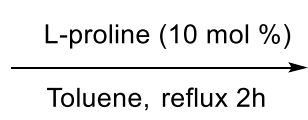

Toluene, reflux $2 \mathrm{~h}$<smiles>Cc1ccc(N2C3=C(C(=O)CC(C)(C)C3)C3(O)c4cccc5cccc(c45)C23O)cc1</smiles><smiles>Cc1ccc(N2C3=C(C(=O)CC(C)(C)C3)C3(O)c4cccc5cccc(c45)C23O)cc1</smiles>

Scheme 2. Preliminary mechanism study. 


\section{Experimental}

\subsection{General Information}

All chemicals were obtained commercially and used without further purification. Melting points were measured using an XT-5 micro melting point apparatus from Beijing Tech Instrument Co., Ltd., (Beijing, China) and are uncorrected. NMR spectra were recorded in DMSO- $d_{6}$ or $\mathrm{CDCl}_{3}$ solution on Inova-300 or $400 \mathrm{MHz}$ spectrometers (Varian, Palo Alto, CA, USA). Chemical shifts values are given in ppm and referred as the internal standard to TMS (tetramethylsilane). The coupling constants $(J)$ are reported in hertz (Hz). High-resolution mass spectra (HRMS) were obtained using a MicrOTOF-Q II instrument from Bruker (Billerica, MA, USA). X-ray crystal diffraction analysis was performed with a Mercury CCD X-ray diffractometer (Rigaku, Akishima, Tokyo, Japan).

\subsection{General Procedure for the Synthesis of Acenaphtho[1,2-b]indole Derivatives 3}

A mixture of enaminone $1(1.0 \mathrm{mmol})$, acenaphthoquinone $(2,1.0 \mathrm{mmol})$, L-proline $(0.1 \mathrm{mmol})$ and toluene $(5 \mathrm{~mL})$ was refluxed for $1-3 \mathrm{~h}$. After the completion of the reaction (confirmed by TLC), the reaction mixture was concentrated in vacuo. Then, acetic acid $(15 \mathrm{~mL})$ and conc. $\mathrm{H}_{2} \mathrm{SO}_{4}(0.5 \mathrm{~mL})$ were added. The reaction mixture was stirred at $80{ }^{\circ} \mathrm{C}$ for $1-2 \mathrm{~h}$. After completion of the reaction (confirmed by TLC), the reaction mixture was then cooled to room temperature and concentrated in vacuo. The crude mixture was purified by column chromatography on silica gel using ethyl acetate/petroleum ether 1:3 as the eluents to give the corresponding product 3.

9,9-Dimethyl-7-(p-tolyl)-9,10-dihydro-7H-acenaphtho[1,2-b]indol-11(8H)-one (3a). White solid, $R_{f}=0.56$, m.p. $214-216^{\circ} \mathrm{C}$. IR $\left(\mathrm{KBr}, \mathrm{cm}^{-1}\right) v$ : 3063, 2951, 1724, 1630, 1504, 1483, 1323, 1259, 1156, 1140, 860, 771, 722, 679. ${ }^{1} \mathrm{H}-\mathrm{NMR}\left(400 \mathrm{MHz}, \mathrm{CDCl}_{3}\right) \delta 8.11(\mathrm{~d}, J=6.4 \mathrm{~Hz}, 1 \mathrm{H}, \mathrm{ArH}), 7.56(\mathrm{t}, J=8.8 \mathrm{~Hz}, 2 \mathrm{H}, \mathrm{ArH}), 7.47$ $(\mathrm{t}, J=6.8 \mathrm{~Hz}, 1 \mathrm{H}, \mathrm{ArH}), 7.35-7.33(\mathrm{~m}, 4 \mathrm{H}, \mathrm{ArH}), 7.21(\mathrm{t}, J=7.6 \mathrm{~Hz}, 1 \mathrm{H}, \mathrm{ArH}), 7.04(\mathrm{~d}, J=6.8 \mathrm{~Hz}, 1 \mathrm{H}$, $\mathrm{ArH}), 2.56\left(\mathrm{~s}, 2 \mathrm{H}, \mathrm{CH}_{2}\right), 2.42\left(\mathrm{~s}, 3 \mathrm{H}, \mathrm{CH}_{3}\right), 2.40\left(\mathrm{~s}, 2 \mathrm{H}, \mathrm{CH}_{2}\right), 1.05\left(\mathrm{~s}, 6 \mathrm{H}, 2 \times \mathrm{CH}_{3}\right) .{ }^{13} \mathrm{C}-\mathrm{NMR}(75 \mathrm{MHz}$, $\left.\mathrm{CDCl}_{3}\right) \delta 192.8,145.5,138.1,137.7,133.7,131.0,130.8,129.3,128.2,128.0,127.1,125.6,125.3,125.0,124.7$, $124.6,122.8,117.9,115.2,51.1,36.1,34.8,27.6,20.3$. HRMS (ESI) $m / z$ : Calcd. for $\mathrm{C}_{27} \mathrm{H}_{23} \mathrm{NONa}[\mathrm{M}+\mathrm{Na}]^{+}$ 400.1677. Found: 400.1705.

7-(3-Chloro-4-fluorophenyl)-9,9-dimethyl-9,10-dihydro-7H-acenaphtho[1,2-b]indol-11(8H)-one (3b). White solid, $R_{f}=0.61$, m.p. $248-250{ }^{\circ} \mathrm{C}$. IR $\left(\mathrm{KBr}, \mathrm{cm}^{-1}\right) v: 3027,2939,1721,1494,1343,1174,895,818 .{ }^{1} \mathrm{H}-\mathrm{NMR}$ $\left(400 \mathrm{MHz}, \mathrm{CDCl}_{3}\right) \delta 8.18(\mathrm{~d}, J=6.8 \mathrm{~Hz}, 1 \mathrm{H}, \mathrm{ArH}), 7.69-7.65(\mathrm{~m}, 3 \mathrm{H}, \mathrm{ArH}), 7.56(\mathrm{t}, J=7.6 \mathrm{~Hz}, 1 \mathrm{H}, \mathrm{ArH})$, 7.44-7.31 (m, 3H, ArH), $7.10(\mathrm{~d}, J=6.8 \mathrm{~Hz}, 1 \mathrm{H}, \mathrm{ArH}), 2.57\left(\mathrm{~s}, 2 \mathrm{H}, \mathrm{CH}_{2}\right), 2.43\left(\mathrm{~s}, 2 \mathrm{H}, \mathrm{CH}_{2}\right), 1.12(\mathrm{~s}, 6 \mathrm{H}, 2$ $\left.\times \mathrm{CH}_{3}\right) .{ }^{13} \mathrm{C}-\mathrm{NMR}\left(75 \mathrm{MHz}, \mathrm{CDCl}_{3}\right) \delta 193.7,156.3,146.2,138.8,131.7,131.6,129.3,128.5,128.2,128.2$, $126.7,126.5,126.3,125.9,125.8,124.2,118.7,117.9,117.6,116.6,51.9,37.0,35.9,28.6$. HRMS (ESI) $m / z$ : Calcd. for $\mathrm{C}_{26} \mathrm{H}_{19} \mathrm{ClFNONa}[\mathrm{M}+\mathrm{Na}]^{+}$438.1037. Found: 438.1020 .

7-(4-Methoxyphenyl)-9,9-dimethyl-9,10-dihydro-7H-acenaphtho[1,2-b]indol-11(8H)-one (3c). White solid, $R_{f}=0.57$, m.p. $240-242{ }^{\circ} \mathrm{C}$. IR $\left(\mathrm{KBr}, \mathrm{cm}^{-1}\right)$ v: 3037, 2944, 1723, 1511, 1443, 1078, 816. ${ }^{1} \mathrm{H}-\mathrm{NMR}(400 \mathrm{MHz}$, $\left.\mathrm{CDCl}_{3}\right) \delta 8.10-8.08(\mathrm{~m}, 1 \mathrm{H}, \mathrm{ArH}), 7.58-7.54(\mathrm{~m}, 2 \mathrm{H}, \mathrm{ArH}), 7.48-7.46(\mathrm{~m}, 1 \mathrm{H}, \mathrm{ArH}), 7.37-7.35(\mathrm{~m}, 2 \mathrm{H}$, ArH), 7.23-7.17 (m, 1H, ArH), 7.01-7.00 (m, 3H, ArH), 3.84-3.83 (m, 3H, $\left.\mathrm{CH}_{3} \mathrm{O}\right), 2.52\left(\mathrm{~s}, 2 \mathrm{H}, \mathrm{CH}_{2}\right), 2.46$ (s, 2H, $\left.\mathrm{CH}_{2}\right), 1.04\left(\mathrm{~s}, 6 \mathrm{H}, 2 \times \mathrm{CH}_{3}\right) .{ }^{13} \mathrm{C}-\mathrm{NMR}\left(75 \mathrm{MHz}, \mathrm{CDCl}_{3}\right) \delta 193.8,159.6,146.8,139.3,132.1,131.8$, 130.0, 129.2, 129.0, 128.1, 127.1, 126.7, 126.3, 125.9, 125.5, 123.7, 118.8, 116.1, 114.9, 55.6, 52.0, 36.9, 35.7, 28.6. HRMS (ESI) $m / z$ : Calcd. for $\mathrm{C}_{27} \mathrm{H}_{23} \mathrm{NO}_{2} \mathrm{Na}[\mathrm{M}+\mathrm{Na}]^{+} 416.1626$. Found: 416.1629 .

7-(4-Bromophenyl)-9,9-dimethyl-9,10-dihydro-7H-acenaphtho[1,2-b]indol-11(8H)-one (3d). White solid, $R_{f}=$ 0.60, m.p. $230-232{ }^{\circ} \mathrm{C}$. IR $\left(\mathrm{KBr}, \mathrm{cm}^{-1}\right)$ v: 3049, 2952, 1652, 1609, 1494, 1079, 819, 769. ${ }^{1} \mathrm{H}-\mathrm{NMR}(400 \mathrm{MHz}$, $\left.\mathrm{CDCl}_{3}\right) \delta 8.17(\mathrm{~d}, J=6.4 \mathrm{~Hz}, 1 \mathrm{H}, \mathrm{ArH}), 7.73-7.71(\mathrm{~m}, 2 \mathrm{H}, \mathrm{ArH}), 7.67-7.62(\mathrm{~m}, 2 \mathrm{H}, \mathrm{ArH}), 7.54(\mathrm{t}, J=$ $7.6 \mathrm{~Hz}, 1 \mathrm{H}, \mathrm{ArH}), 7.41-7.39(\mathrm{~m}, 2 \mathrm{H}, \mathrm{ArH}), 7.29(\mathrm{t}, J=7.2 \mathrm{~Hz}, 1 \mathrm{H}, \mathrm{ArH}), 7.10(\mathrm{~d}, J=6.8 \mathrm{~Hz}, 1 \mathrm{H}, \mathrm{ArH})$, $2.55\left(\mathrm{~s}, 2 \mathrm{H}, \mathrm{CH}_{2}\right), 2.41\left(\mathrm{~s}, 2 \mathrm{H}, \mathrm{CH}_{2}\right), 1.10\left(\mathrm{~s}, 6 \mathrm{H}, 2 \times \mathrm{CH}_{3}\right) .{ }^{13} \mathrm{C}-\mathrm{NMR}\left(75 \mathrm{MHz}, \mathrm{CDCl}_{3}\right) \delta$ 193.7, 146.2, 138.6, 136.3, 133.0, 131.8, 131.7, 129.3, 128.7, 128.2, 127.4, 126.7, 126.5, 126.2, 126.1, 124.0, 122.4, 118.9, 
116.5, 51.9, 37.0, 35.8, 28.6. HRMS (ESI) $m / z$ : Calcd. for $\mathrm{C}_{26} \mathrm{H}_{20} \mathrm{BrNONa}[\mathrm{M}+\mathrm{Na}]^{+}$464.0626. Found: 464.0633 .

9,9-Dimethyl-7-(4-nitrophenyl)-9,10-dihydro-7H-acenaphtho[1,2-b]indol-11(8H)-one (3e). Yellow solid, $R_{f}=0.63$, m.p. $240-242{ }^{\circ} \mathrm{C} . \mathrm{IR}\left(\mathrm{KBr}^{\mathrm{cm}}{ }^{-1}\right) v: 3036,2953,2350,1728,1592,1505,1329,1068,842$. ${ }^{1} \mathrm{H}-\mathrm{NMR}\left(400 \mathrm{MHz}, \mathrm{CDCl}_{3}\right) \delta 8.60(\mathrm{~d}, J=7.2 \mathrm{~Hz}, 1 \mathrm{H}, \mathrm{ArH}), 8.52(\mathrm{~d}, J=8.4 \mathrm{~Hz}, 1 \mathrm{H}, \mathrm{ArH}), 8.31-8.26$ $(\mathrm{m}, 3 \mathrm{H}, \mathrm{ArH}), 8.09(\mathrm{~d}, J=6.8 \mathrm{~Hz}, 1 \mathrm{H}, \mathrm{ArH}), 7.84-7.80(\mathrm{~m}, 3 \mathrm{H}, \mathrm{ArH}), 7.67(\mathrm{~d}, J=8.0 \mathrm{~Hz}, 1 \mathrm{H}, \mathrm{ArH}), 2.72$ $\left(\mathrm{s}, 1 \mathrm{H}, \mathrm{CH}_{2}\right), 2.51\left(\mathrm{~s}, 1 \mathrm{H}, \mathrm{CH}_{2}\right), 2.17\left(\mathrm{~s}, 1 \mathrm{H}, \mathrm{CH}_{2}\right), 1.64\left(\mathrm{~s}, 1 \mathrm{H}, \mathrm{CH}_{2}\right), 1.25\left(\mathrm{~s}, 3 \mathrm{H}, \mathrm{CH}_{3}\right), 1.16\left(\mathrm{~s}, 3 \mathrm{H}, \mathrm{CH}_{3}\right)$. ${ }^{13} \mathrm{C}-\mathrm{NMR}\left(75 \mathrm{MHz}, \mathrm{CDCl}_{3}\right) \delta 187.1,159.5,146.1,145.0,141.8,134.3,132.4,131.6,127.5,127.4,126.4$, 125.8, 125.6, 125.5, 125.3, 124.4, 123.4, 121.0, 117.9, 117.7, 50.9, 35.0, 28.7, 27.6. HRMS (ESI) $\mathrm{m} / z$ : Calcd. for $\mathrm{C}_{26} \mathrm{H}_{20} \mathrm{~N}_{2} \mathrm{O}_{3} \mathrm{Na}[\mathrm{M}+\mathrm{Na}]^{+}$431.1372. Found: 431.1355 .

7-(3,5-Dimethylphenyl)-9,9-dimethyl-9,10-dihydro-7H-acenaphtho[1,2-b]indol-11(8H)-one (3f). White solid, $R_{f}=0.57$, m.p. $220-221^{\circ} \mathrm{C} . \mathrm{IR}\left(\mathrm{KBr}, \mathrm{cm}^{-1}\right) v: 3076,2956,1728,1638,1510,1474,1383,1181,1080,870$, 836, 801, 767. ${ }^{1} \mathrm{H}-\mathrm{NMR}\left(400 \mathrm{MHz}, \mathrm{CDCl}_{3}\right) \delta 8.18(\mathrm{~d}, J=6.4 \mathrm{~Hz}, 1 \mathrm{H}, \mathrm{ArH}), 7.66-7.62(\mathrm{~m}, 2 \mathrm{H}, \mathrm{ArH})$, $7.55(\mathrm{t}, J=7.2 \mathrm{~Hz}, 1 \mathrm{H}, \mathrm{ArH}), 7.30(\mathrm{t}, J=7.6 \mathrm{~Hz}, 1 \mathrm{H}, \mathrm{ArH}), 7.18-7.15(\mathrm{~m}, 3 \mathrm{H}, \mathrm{ArH}), 7.11(\mathrm{~d}, J=6.8 \mathrm{~Hz}$, $1 \mathrm{H}, \mathrm{ArH}), 2.65\left(\mathrm{~s}, 2 \mathrm{H}, \mathrm{CH}_{2}\right), 2.48\left(\mathrm{~s}, 2 \mathrm{H}, \mathrm{CH}_{2}\right), 2.46\left(\mathrm{~s}, 6 \mathrm{H}, 2 \times \mathrm{CH}_{3}\right), 1.15\left(\mathrm{~s}, 6 \mathrm{H}, 2 \times \mathrm{CH}_{3}\right) .{ }^{13} \mathrm{C}-\mathrm{NMR}$ $\left(75 \mathrm{MHz}, \mathrm{CDCl}_{3}\right) \delta 193.9,146.5,139.7,139.1,137.2,132.1,130.3,129.2,129.1,128.1,126.7,126.3,126.0$, $123.8,123.4,118.9,116.2,52.1,37.2,35.9,28.6,21.4$. HRMS (ESI) $m / z$ : Calcd. for $\mathrm{C}_{28} \mathrm{H}_{25} \mathrm{NONa}[\mathrm{M}+$ $\mathrm{Na}]^{+}$414.1834. Found: 414.1847 .

9,9-Dimethyl-7-phenyl-9,10-dihydro-7H-acenaphtho[1,2-b]indol-11(8H)-one (3g). White solid, $R_{f}=0.55$, m.p. 200-202 ${ }^{\circ} \mathrm{C}$. IR $\left(\mathrm{KBr}, \mathrm{cm}^{-1}\right) v: 3042,2958,1649,1520,1500,1394,1081,821,774,706 .{ }^{1} \mathrm{H}-\mathrm{NMR}(400 \mathrm{MHz}$, $\left.\mathrm{CDCl}_{3}\right) \delta 8.13(\mathrm{~d}, J=6.0 \mathrm{~Hz}, 1 \mathrm{H}, \mathrm{ArH}), 7.60-7.50(\mathrm{~m}, 8 \mathrm{H}, \mathrm{ArH}), 7.23(\mathrm{dd}, J=14.0,6.8 \mathrm{~Hz}, 1 \mathrm{H}, \mathrm{ArH}), 7.05$ $(\mathrm{d}, J=6.4 \mathrm{~Hz}, 1 \mathrm{H}, \mathrm{ArH}), 2.59\left(\mathrm{~s}, 2 \mathrm{H}, \mathrm{CH}_{2}\right), 2.42\left(\mathrm{~s}, 2 \mathrm{H}, \mathrm{CH}_{2}\right), 1.09\left(\mathrm{~s}, 6 \mathrm{H}, 2 \times \mathrm{CH}_{3}\right) .{ }^{13} \mathrm{C}-\mathrm{NMR}(75 \mathrm{MHz}$, $\left.\mathrm{CDCl}_{3}\right) \delta 193.8,146.5,138.9,137.3,132.0,131.8,129.8,129.2,128.9,128.7,128.1,126.7,126.3,126.1,125.8$, 123.8, 118.9, 116.3, 52.0, 37.0, 35.8, 28.6. HRMS (ESI) $\mathrm{m} / z$ : Calcd. for $\mathrm{C}_{26} \mathrm{H}_{21} \mathrm{NONa}[\mathrm{M}+\mathrm{Na}]^{+} 386.1521$. Found: 386.1503 .

7-(2-Chlorophenyl)-9,9-dimethyl-9,10-dihydro-7H-acenaphtho[1,2-b]indol-11(8H)-one (3h). White solid, $R_{f}=0.59$, m.p. $210-212{ }^{\circ} \mathrm{C}$. IR $\left(\mathrm{KBr}, \mathrm{cm}^{-1}\right) v: 3041,2957,1651,1518,1491,1458,1069,821,771$. ${ }^{1} \mathrm{H}-\mathrm{NMR}\left(400 \mathrm{MHz}, \mathrm{CDCl}_{3}\right) \delta 8.15(\mathrm{~d}, J=6.8 \mathrm{~Hz}, 1 \mathrm{H}, \mathrm{ArH}), 7.67-7.59(\mathrm{~m}, 3 \mathrm{H}, \mathrm{ArH}), 7.53-7.49(\mathrm{~m}, 4 \mathrm{H}$, $\mathrm{ArH}), 7.23(\mathrm{~d}, J=6.4 \mathrm{~Hz}, 1 \mathrm{H}, \mathrm{ArH}), 6.76(\mathrm{~d}, J=6.8 \mathrm{~Hz}, 1 \mathrm{H}, \mathrm{ArH}), 2.52-2.46\left(\mathrm{~m}, 4 \mathrm{H}, 2 \times \mathrm{CH}_{2}\right), 1.14-1.11$ $\left(\mathrm{m}, 6 \mathrm{H}, 2 \times \mathrm{CH}_{3}\right) .{ }^{13} \mathrm{C}-\mathrm{NMR}\left(75 \mathrm{MHz}, \mathrm{CDCl}_{3}\right) \delta 193.8,147.5,139.5,135.1,132.3,132.1,131.7,131.0$, 130.8, 129.3, 129.2, 128.7, 128.2, 128.1, 126.7, 126.4, 126.0, 125.5, 124.0, 118.4, 116.2, 52.2, 36.4, 35.9, 29.0, 28.2. HRMS (ESI) $m / z$ : Calcd. for $\mathrm{C}_{26} \mathrm{H}_{20} \mathrm{ClNONa}[\mathrm{M}+\mathrm{Na}]^{+} 420.1131$. Found: 420.1169 .

7-(4-(tert-Butyl)phenyl)-9,9-dimethyl-9,10-dihydro-7H-acenaphtho[1,2-b]indol-11(8H)-one (3i). White solid, $R_{f}=0.56$, m.p. $250-252{ }^{\circ} \mathrm{C}$. IR $\left(\mathrm{KBr}, \mathrm{cm}^{-1}\right) v: 3021,2940,1700,1452,1339,1057,893,767 .{ }^{1} \mathrm{H}-\mathrm{NMR}$ $\left(400 \mathrm{MHz}, \mathrm{CDCl}_{3}\right) \delta 8.18(\mathrm{~d}, J=5.6 \mathrm{~Hz}, 1 \mathrm{H}, \mathrm{ArH}), 7.64-7.54(\mathrm{~m}, 5 \mathrm{H}, \mathrm{ArH}), 7.47-7.45(\mathrm{~m}, 2 \mathrm{H}, \mathrm{ArH})$, 7.31-7.29 (t, J = 4.8 Hz, 1H, ArH), $7.13(\mathrm{~d}, J=6.0 \mathrm{~Hz}, 1 \mathrm{H}, \mathrm{ArH}), 2.67\left(\mathrm{~s}, 2 \mathrm{H} \mathrm{CH}_{2}\right), 2.48\left(\mathrm{~s}, 2 \mathrm{H}, \mathrm{CH}_{2}\right), 1.44$ $\left(\mathrm{s}, 9 \mathrm{H}, \mathrm{C}\left(\mathrm{CH}_{3}\right)_{3}\right), 1.14\left(\mathrm{~s}, 6 \mathrm{H}, 2 \times \mathrm{CH}_{3}\right) .{ }^{13} \mathrm{C}-\mathrm{NMR}\left(75 \mathrm{MHz}, \mathrm{CDCl}_{3}\right) \delta 193.8,151.8,146.6,139.0,134.7$, 132.0, 131.9, 129.2, 129.1, 128.1, 126.6, 126.3, 126.0, 125.2, 123.8, 119.0, 116.3, 52.1, 37.2, 35.8, 34.9, 31.4, 28.6. HRMS (ESI) $m / z$ : Calcd. for $\mathrm{C}_{30} \mathrm{H}_{28} \mathrm{NO}[\mathrm{M}-\mathrm{H}]^{+}$418.2171. Found: 418.2147.

7-Phenyl-9,10-dihydro-7H-acenaphtho[1,2-b]indol-11(8H)-one (3j). White solid, $R_{f}=0.57$, m.p. 208-210 ${ }^{\circ} \mathrm{C}$. IR $\left(\mathrm{KBr}, \mathrm{cm}^{-1}\right)$ v: 3038, 2956, 1720, 1698, 1498, 1341, 1136, 837, 734. ${ }^{1} \mathrm{H}-\mathrm{NMR}\left(400 \mathrm{MHz}, \mathrm{CDCl}_{3}\right) \delta 8.10$ $(\mathrm{d}, J=6.0 \mathrm{~Hz}, 1 \mathrm{H}, \mathrm{ArH}), 7.56-7.43(\mathrm{~m}, 8 \mathrm{H}, \mathrm{ArH}), 7.18(\mathrm{~d}, J=7.2 \mathrm{~Hz}, 1 \mathrm{H}, \mathrm{ArH}), 7.01(\mathrm{~d}, J=6.0 \mathrm{~Hz}, 1 \mathrm{H}$, $\mathrm{ArH}), 2.67\left(\mathrm{~s}, 2 \mathrm{H}, \mathrm{CH}_{2}\right), 2.51\left(\mathrm{~s}, 2 \mathrm{H}, \mathrm{CH}_{2}\right), 2.07\left(\mathrm{~s}, 2 \mathrm{H}, \mathrm{CH}_{2}\right) .{ }^{13} \mathrm{C}-\mathrm{NMR}\left(75 \mathrm{MHz}, \mathrm{CDCl}_{3}\right) \delta$ 194.4, 147.6, 138.7, 137.4, 132.0, 131.9, 129.8, 129.2, 128.9, 128.6, 128.1, 126.6, 126.4, 126.1, 126.0, 125.7, 123.9, 119.0, 117.4, 38.1, 24.0, 23.3. HRMS(ESI) m/z: Calcd. for $\mathrm{C}_{24} \mathrm{H}_{16} \mathrm{NO}[\mathrm{M}-\mathrm{H}]^{+}$334.1232. Found 334.1234.

7-(2-Chlorophenyl)-9,10-dihydro-7H-acenaphtho[1,2-b]indol-11(8H)-one (3k). White solid, $R_{f}=0.39$, m.p. 193-194 ${ }^{\circ} \mathrm{C}$. IR $\left(\mathrm{KBr}, \mathrm{cm}^{-1}\right)$ v: 3051, 2943, 1723, 1656, 1522, 1072, 820, 773, 745. ${ }^{1} \mathrm{H}-\mathrm{NMR}(400 \mathrm{MHz}$, 
$\left.\mathrm{CDCl}_{3}\right) \delta 8.10(\mathrm{~d}, J=6.4 \mathrm{~Hz}, 1 \mathrm{H}, \mathrm{ArH}), 7.55-7.51(\mathrm{~m}, 3 \mathrm{H}, \mathrm{ArH}), 7.47-7.38(\mathrm{~m}, 4 \mathrm{H}, \mathrm{ArH}), 7.16(\mathrm{t}, J=7.2 \mathrm{~Hz}$, $1 \mathrm{H}, \mathrm{ArH}), 6.71(\mathrm{~d}, J=6.8 \mathrm{~Hz}, 1 \mathrm{H}, \mathrm{ArH}), 2.61-2.50\left(\mathrm{~m}, 4 \mathrm{H}, 2 \times \mathrm{CH}_{2}\right), 2.09-2.05\left(\mathrm{~m}, 2 \mathrm{H}, \mathrm{CH}_{2}\right) .{ }^{13} \mathrm{C}-\mathrm{NMR}$ $\left(75 \mathrm{MHz}, \mathrm{CDCl}_{3}\right) \delta 194.4,148.6,139.3,135.0,132.1,131.7,131.0,130.8,129.2,129.1,128.7,128.2,128.1$, 126.7, 126.4, 126.0, 125.7, 123.9, 118.5, 117.4, 38.1, 23.8, 22.6. HRMS (ESI) $m / z$ : Calcd. for $\mathrm{C}_{24} \mathrm{H}_{16} \mathrm{ClNONa}$ $[\mathrm{M}+\mathrm{Na}]^{+}$392.0818. Found: 392.0830 .

7-(2,4-Dimethylphenyl)-9,10-dihydro-7H-acenaphtho[1,2-b]indol-11(8H)-one (31). White solid, $R_{f}=0.35$, m.p. 264-267 ${ }^{\circ} \mathrm{C}$. IR $\left(\mathrm{KBr}, \mathrm{cm}^{-1}\right)$ v: 3037, 2943, 1724, 1690, 1461, 1337, 1156, 788. ${ }^{1} \mathrm{H}-\mathrm{NMR}\left(400 \mathrm{MHz}, \mathrm{CDCl}_{3}\right)$ $\delta$ 8.21-8.19 (m, 1H, ArH), 7.66-7.56 (m, 5H, ArH), 7.51-7.44 (m, 2H, ArH), $7.31(\mathrm{t}, 1 \mathrm{H}, J=4.8 \mathrm{~Hz}, \mathrm{ArH})$, $7.15(\mathrm{~d}, J=6.0 \mathrm{~Hz}, 1 \mathrm{H}, \mathrm{ArH}), 2.84-2.79\left(\mathrm{~m}, 2 \mathrm{H}, \mathrm{CH}_{2}\right), 2.64-2.58\left(\mathrm{~m}, 2 \mathrm{H}, \mathrm{CH}_{2}\right), 2.22-2.15\left(\mathrm{~m}, 2 \mathrm{H}, \mathrm{CH}_{2}\right)$, $1.42\left(\mathrm{~s}, 9 \mathrm{H}, 3 \times \mathrm{CH}_{3}\right) .{ }^{13} \mathrm{C}-\mathrm{NMR}\left(75 \mathrm{MHz}, \mathrm{CDCl}_{3}\right) \delta 194.5,151.8,147.8,138.7,134.6,132.1,131.9,129.2$, 129.0, 128.1, 126.6, 126.3, 126.0, 125.1, 123.8, 119.1, 117.3, 38.1, 34.9, 31.4, 24.0, 23.4. HRMS calcd for $\mathrm{C}_{28} \mathrm{H}_{25} \mathrm{NONa}[\mathrm{M}+\mathrm{Na}]^{+} 414.1834$, found 414.1834 .

7-(4-(tert-Butyl)phenyl)-9,10-dihydro-7H-acenaphtho[1,2-b]indol-11(8H)-one (3m). White solid, $R_{f}=0.36$, m.p. $190-192{ }^{\circ} \mathrm{C}$. IR $\left(\mathrm{KBr}, \mathrm{cm}^{-1}\right)$ v: 3049, 2953, 1655, 1521, 1082, 819, 773. ${ }^{1} \mathrm{H}-\mathrm{NMR}\left(400 \mathrm{MHz}, \mathrm{CDCl}_{3}\right)$ $\delta 8.17(\mathrm{~d}, J=6.4 \mathrm{~Hz}, 1 \mathrm{H}, \operatorname{ArH}), 7.64-7.59(\mathrm{~m}, 2 \mathrm{H}, \operatorname{ArH}), 7.53(\mathrm{t}, J=7.2 \mathrm{~Hz}, 1 \mathrm{H}, \operatorname{ArH}), 7.25-7.22(\mathrm{~m}$, $3 \mathrm{H}, \mathrm{ArH}), 7.17(\mathrm{~d}, J=8.0 \mathrm{~Hz}, 1 \mathrm{H}, \mathrm{ArH}), 6.76(\mathrm{~d}, J=6.8 \mathrm{~Hz}, 1 \mathrm{H}, \mathrm{ArH}), 2.67\left(\mathrm{t}, J=10.4 \mathrm{~Hz}, 1 \mathrm{H}, \mathrm{CH}_{2}\right)$, 2.62-2.59 (m, 2H, $\left.\mathrm{CH}_{2}\right), 2.53\left(\mathrm{t}, J=5.6 \mathrm{~Hz}, 1 \mathrm{H}, \mathrm{CH}_{2}\right), 2.44\left(\mathrm{~s}, 3 \mathrm{H}, \mathrm{CH}_{3}\right), 2.18-2.15\left(\mathrm{~m}, 2 \mathrm{H}, \mathrm{CH}_{2}\right), 2.07$ $\left(\mathrm{s}, 3 \mathrm{H}, \mathrm{CH}_{3}\right) .{ }^{13} \mathrm{C}-\mathrm{NMR}\left(75 \mathrm{MHz}, \mathrm{CDCl}_{3}\right) \delta 194.3,148.1,139.5,135.2,133.7,132.3,132.1,131.8,129.2$, 129.0, 128.2, 127.9, 127.2, 126.7, 126.2, 125.8, 123.7, 118.3, 117.0, 38.2, 24.0, 22.6, 21.3, 17.4. HRMS (ESI) $m / z$ : Calcd. for $\mathrm{C}_{26} \mathrm{H}_{21} \mathrm{NONa}[\mathrm{M}+\mathrm{Na}]^{+}$386.1521. Found: 386.1566 .

7-(3,5-Dimethylphenyl)-9,10-dihydro-7H-acenaphtho[1,2-b]indol-11(8H)-one (3n). White solid, $R_{f}=0.34$, m.p. 222-224. IR $\left(\mathrm{KBr}, \mathrm{cm}^{-1}\right)$ v: 3043, 2940, 1721, 1660, 1521, 1071, 1034, 852, 815, 767. ${ }^{1} \mathrm{H}-\mathrm{NMR}$ (400 MHz, $\left.\mathrm{CDCl}_{3}\right) \delta 8.19-8.17(\mathrm{~m}, 1 \mathrm{H}, \mathrm{ArH}), 7.66-7.62(\mathrm{~m}, 2 \mathrm{H}, \mathrm{ArH}), 7.56-7.51(\mathrm{~m}, 1 \mathrm{H}, \mathrm{ArH}), 7.31-7.28$ $(\mathrm{m}, 1 \mathrm{H}, \mathrm{ArH}), 7.17-7.11(\mathrm{~m}, 4 \mathrm{H}, \mathrm{ArH}), 2.81\left(\mathrm{t}, J=5.2 \mathrm{~Hz}, 2 \mathrm{H}, \mathrm{CH}_{2}\right), 2.63-2.59\left(\mathrm{~m}, 2 \mathrm{H}, \mathrm{CH}_{2}\right), 2.44(\mathrm{~s}, 6 \mathrm{H}$, $\left.2 \times \mathrm{CH}_{3}\right), 2.19\left(\mathrm{t}, J=5.6 \mathrm{~Hz}, 2 \mathrm{H}, \mathrm{CH}_{2}\right) .{ }^{13} \mathrm{C}-\mathrm{NMR}\left(75 \mathrm{MHz}, \mathrm{CDCl}_{3}\right) \delta 194.5,147.7,139.6,138.8,137.2$, 132.1, 131.9, 130.2, 129.2, 129.1, 128.1, 126.7, 126.3, 126.0, 123.8, 123.3, 118.9, 117.3, 38.1, 24.0, 23.4, 21.4 . HRMS (ESI) $m / z$ : Calcd. for $\mathrm{C}_{26} \mathrm{H}_{20} \mathrm{NO}[\mathrm{M}-\mathrm{H}]^{+}$363.1545. Found: 363.1557.

\subsection{General Procedure for the Synthesis of Tetrahydroacenaphtho[1,2-b]indole Derivatives Fa}

A mixture of enaminone (1a) $(1.0 \mathrm{mmol})$, acenaphthoquinone (2) $(1.0 \mathrm{mmol})$, L-proline $(0.1 \mathrm{mmol})$ and toluene $(5 \mathrm{~mL})$ was refluxed for $3 \mathrm{~h}$. After the completion of the reaction (confirmed by TLC), the reaction mixture was concentrated in vacuo. The crude mixture was purified by column chromatography on silica gel using ethyl acetate/petroleum ether 1:1 as the eluents to give corresponding product Fa.

6b,11b-Dihydroxy-9,9-dimethyl-7-(p-tolyl)-8,9,10,11b-tetrahydro-6bH-acenaphtho[1,2-b]indol-11(7H)-one (Fa). White solid, $R_{f}=0.23$, m.p. $240-242{ }^{\circ} \mathrm{C}$. IR $\left(\mathrm{KBr}, \mathrm{cm}^{-1}\right) v: 3598,2961,2870,1790,1606,1511,1436,1406$, 1283, 1038, 783. ${ }^{1} \mathrm{H}$ NMR (300 MHz, DMSO- $\left.d_{6}\right) \delta 7.94-6.93(\mathrm{~m}, 10 \mathrm{H}, \mathrm{ArH}), 6.46(\mathrm{~s}, 1 \mathrm{H}, \mathrm{OH}), 5.80(\mathrm{~s}$, $1 \mathrm{H}, \mathrm{OH}), 2.38-1.79\left(\mathrm{~m}, 7 \mathrm{H}, \mathrm{CH}_{3}+2 \times \mathrm{CH}_{2}\right), 1.01\left(\mathrm{~s}, 3 \mathrm{H}, \mathrm{CH}_{3}\right), 0.83\left(\mathrm{~s}, 3 \mathrm{H}, \mathrm{CH}_{3}\right) .{ }^{13} \mathrm{C} \mathrm{NMR}(100 \mathrm{MHz}$, DMSO-d $\left.d_{6}\right) \delta 194.5,168.6,149.7,145.7,142.4,140.9,139.8,136.0,135.0,134.9,134.2,133.8,132.7,130.1$, 128.6, 126. 6, 124.8, 115.9, 107.8, 91.9, 56.3, 42.3, 38.7, 34.7, 32.3, 26.2. HRMS (ESI) $m / z$ : Calcd. for $\mathrm{C}_{27} \mathrm{H}_{25} \mathrm{NO}_{3} \mathrm{Na}[\mathrm{M}+\mathrm{Na}]^{+}$434.1732. Found: 434.1734.

\section{Conclusions}

In summary, we have developed an efficient protocol for the construction of acenaphtho[1,2-b]indole derivatives via the domino reaction of enaminones with acenaphthoquinone catalyzed by L-proline. This protocol has the advantages of mild reaction conditions, high yields and operational convenience. 
Supplementary Materials: Copies of the ${ }^{1} \mathrm{H}-\mathrm{NMR}$ and ${ }^{13} \mathrm{C}-\mathrm{NMR}$ spectra of the compounds are available in the online Supplementary Materials.

Author Contributions: J.W. and Y.W. conceived and designed the experiments. G.-N.Z., X.Y. and M.Z. performed the experiments. W.N. performed the experiments supporting the proposed reaction mechanism. G.-N.Z. wrote the manuscript with the help of J.W. and Y.W. All authors read and approved the final manuscript.

Funding: Financial support for this research provided by the National Natural Science Foundation of China (81473098, 81473099, and 81703366) and the CAMS Innovation Fund for Medical Sciences (2016-I2M-3-014, 2016-I2M-1-011, and 2017-I2M-3-019) is gratefully acknowledged.

Conflicts of Interest: The authors declare no conflict of interest.

\section{References}

1. Somei, M.; Yamada, F. Simple indole alkaloids and those with a nonrearranged monoterpenoid unit. Nat. Prod. Rep. 2004, 21, 278-311. [CrossRef] [PubMed]

2. Bandidni, M.; Eichholzer, A. Catalytic functionalization of indoles in a new dimension. Angew. Chem. Int. Ed. 2009, 48, 9608-9644. [CrossRef] [PubMed]

3. Kochanowska-Karamya, A.J.; Hamann, M.J. Marine indole alkaloids: Potential new drug leads for the control of depression and anxiety. Chem. Rev. 2010, 110, 4480-4497. [CrossRef]

4. Chen, I.; Safe, S.; Bjeldanes, L. Indole-3-carbinol and diindolymethane as aryl hydrocarbon (Ah) receptor agonists and antagonists in T47D human breast cancer. Biochem. Pharmacol. 1996, 51, 1069-1076. [CrossRef]

5. Suzen, S.; Buyukbingol, E. Anti-cancer activity studies of indolalthiohydantoin (PTT) on certain cancer cell lines. Farmaco 2000, 55, 246-248. [CrossRef]

6. Giagoudakis, G.; Markantonis, S.L. Relationships between the concentrations of prostaglandins and the nonsteroidal anti-inflammatory drugs indomethacin, diclofenac, and ibuprofen. Pharmacotherapy 2005, 25, 18-25. [CrossRef] [PubMed]

7. Suzen, S.; Buyukbingol, E. Evaluation of anti-HIV activity of 5-(2-phenyl-3'-indolal)-2-thiohydantoin. Farmaco 1998, 53, 525-527. [CrossRef]

8. Walter, G.; Liebl, R.; voa Angerer, E. 2-Phenylindole sulfamates: Inhibitors of steroid sulfatase with antiproliferative activity in MCF-7 breast cancer cells. J. Steroid Biochem. Mol. Biol. 2004, 88, 409-420. [CrossRef] [PubMed]

9. Ge, X.; Yannai, S.; Rennert, G.; Gruener, N.; Fares, F.A. 3,3'-Diindolymethane induces apoptosisin human cancer cells. Biochem. Biophys. Res. Commun. 1996, 228, 153-158. [CrossRef] [PubMed]

10. Horton, D.A.; Bourne, G.T.; Smythe, M.L. The combinational synthesis of bicyclic privileged structures or privileged substructures. Chem. Rev. 2003, 103, 893-930. [CrossRef] [PubMed]

11. Shiri, M. Indoles in multicomponent process (MCPs). Chem. Rev. 2012, 112, 3508-3549. [CrossRef] [PubMed]

12. Lescot, E.; Muzard, G.; Markovits, J.; Belleney, J.; Roques, B.P.; Lepecq, J.B. Synthesis of 11H-pyridocarbazoles and derivatives. Comparison of their DNA binding and antitumor activity with those of $6 \mathrm{H}-$ and 7H-pyridocarbazoles. J. Med. Chem. 1986, 29, 1731-1737. [CrossRef] [PubMed]

13. Thevissen, K.; Marchand, A.; Chaltin, P.; Meert, E.M.K.; Cammue, B.P.A. Antifungal carbazoles. Curr. Med. Chem. 2009, 16, 2205-2211. [CrossRef] [PubMed]

14. Scopton, A.; Kelly, T.R. Synthesis of HKI 0231B. J. Org. Chem. 2005, 70, 10004-10012. [CrossRef] [PubMed]

15. Kraus, A.; Wu, T. A concise synthesis of 5-demethyl-HKI 0231A and 5-demethyl-HKI 0231B. Tetrahedron Lett. 2006, 47, 7801-7804. [CrossRef]

16. Chen, X.B.; Luo, T.B.; Gou, G.Z.; Wang, J.; Liu, W.; Lin, J. Selective synthesis of acenaphtho[1,2-b]indole derivatives via tandem regioselective aza-ene addition/N-cycliaztion/ SN1 type reaction. Asian J. Org. Chem. 2015, 4, 921-928. [CrossRef]

17. Shan, D.; Gao, Y.; Jia, Y. Intramolecular larock indole synthesis: Preparation of 3,4-fused tricyclic indoles and total synthesis of fargesine. Angew. Chem. Int. Ed. 2013, 52, 4902-4905. [CrossRef] [PubMed]

18. Yan, H.; Wang, H.; Li, X.; Xin, X.; Wang, C.; Wan, B. Rhodium-catalyzed C-H annulation of nitrones with alkynes: A regiospecific route to unsymmetrical 2,3-disryl-substituted indoles. Angew. Chem. Int. Ed. 2015, 54, 10613-10617. [CrossRef] [PubMed]

19. Narayanaiyer, V.; Ninadnamdeo, R.; Dilip, H.G. Reaction of dimedone enamines with $\alpha$-ketoacids. J. Chem. Res. (S) 1985, 244-245. [CrossRef] 
20. Shibata, N.; Fujimoto, H.; Mizuta, S.; Ogawa, S.; Ishiuchi, Y.; Nakamura, S.; Toru, T. Efficient synthesis of bicyclic $\alpha$-hydroxy- $\alpha$-trifluoromethyl- $\gamma$-lactams. Synlett 2006, 3484-3488. [CrossRef]

21. Pei, Q.L.; Cui, B.D.; Han, W.Y.; Wu, Z.J.; Zhang, X.M. A facile synthesis of 3-hydroxy-3-(trifluoromethyl)$1 \mathrm{H}$-pyrrol-2(3H) - Ones with BrØnsted acid-catalyzed condensation-cyclization reactions of $\beta$-enamino esters and ethyl trifluoropyruvate. Tetrahedron 2014, 70, 4595-4601. [CrossRef]

22. Tietze, L.F. Domino reactions in organic synthesis. Chem. Rev. 1996, 96, 115-136. [CrossRef] [PubMed]

23. Zubarev, A.A.; Larionova, N.A.; Rodinovskaya, L.A.; Mortikov, V.Y.; Shestopalov, A.M. Synthesis of 2,5-asymmetrically substituted 3,4-diaminothieno[2,3-b]thiophenes by domino reaction. ACS Comb. Sci. 2013, 15, 546-550. [CrossRef] [PubMed]

24. Feng, X.; Wang, J.J.; Zhang, J.J.; Cao, C.P.; Huang, Z.B.; Shi, D.Q. Regioselective synthesis of functionalized $[1,8]$ naphthyridine derivatives via three-component domino reaction under catalyst-free conditions. Green Chem. 2015, 17, 973-981. [CrossRef]

25. Fu, L.; Feng, X.; Zhang, J.J.; Hu, J.D.; Xun, Z.; Wang, J.J.; Huang, Z.B.; Shi, D.Q. Highly efficient construction of a bridged pentacyclic skeleton via a six-component domino reaction under microwave irradiation. Green Chem. 2015, 17, 1535-1545. [CrossRef]

26. Xun, Z.; Feng, X.; Wang, J.J.; Shi, D.Q.; Huang, Z.B. Multicomponent strategy for the preparation of pyrrolo[1,2-a]pyrimidine derivatives under catalyst-free and microwave irradiation conditions. Chin. J. Chem. 2016, 34, 696-702. [CrossRef]

27. Lin, W.; Hu, X.X.; Wang, Y.Z.; Song, S.; Zhang, M.Y.; Shi, D.Q. Microwave-assisted synthesis of 3-substituted indole derivatives via three-component domino reaction. Chin. J. Org. Chem. 2018, 38, 855-862. [CrossRef]

28. Hu, J.D.; Cao, C.P.; Lin, W.; Hu, M.H.; Huang, Z.B.; Shi, D.Q. Selective synthesis of polyfunctionalized pyrido[2,3-b]indoles by multicomponent domino reactions. J. Org. Chem. 2014, 79, 7935-7944. [CrossRef] [PubMed]

29. Cao, C.P.; Xu, C.L.; Lin, W.; Li, X.M.; Hu, M.H.; Wang, J.X.; Huang, Z.B.; Shi, D.Q.; Wang, Y.C. Microwave-assisted improved synthesis of pyrrolo[2,3,4-kl]acridine and dihydropyrrolo[2,3,4-kl]acridine derivatives catalyzed by silica sulfuric acid. Molecules 2013, 18, 1613-1625. [CrossRef] [PubMed]

30. Yang, J.M.; Li, Q.; Zhang, J.J.; Lin, W.; Wang, J.X.; Wang, Y.C.; Huang, Z.B.; Shi, D.Q. Ultrasound-promoted one-pot, four-component synthesis of pyridine-2(1H)-one derivatives. Molecules 2013, 18, 14519-14528. [CrossRef] [PubMed]

31. Wang, J.X.; Zhang, J.J.; Zhao, Y.; Zhang, G.N.; Wang, Y.C.; Shi, D.Q. An efficient and multi-component synthesis of functionalized pyrazole derivatives. Heterocycles 2017, 94, 531-540. [CrossRef]

32. Wang, J.X.; Gao, Y.; Zhang, J.J.; Zhang, G.N.; Ren, J.F.; Zhao, Y.; Wang, Y.C.; Shi, D.Q. An efficient and multi-component synthesis of 5-imino-3,5-dihydro-2H-chromeno[3,4-c] pyridine-2-one derivatives. Heterocycles 2017, 94, 1143-1151. [CrossRef]

Sample Availability: Samples of compounds $\mathbf{3 a}-\mathbf{3 n}$ are available from the authors.

(C) 2018 by the authors. Licensee MDPI, Basel, Switzerland. This article is an open access article distributed under the terms and conditions of the Creative Commons Attribution (CC BY) license (http:/ / creativecommons.org/licenses/by/4.0/). 